\title{
COVID Induced Functional Exhaustion and Persistently Reduced Lymphocytes as Vital Contributing Factors for Post-COVID Rhino-orbital and Cerebral Mucormycosis in Patients with Diabetes: Report from the Indian Sub-continent
}

\author{
Darpan Bhargava $^{1,2}$ (1) $\cdot$ Rajkumar Ahirwal ${ }^{3} \cdot$ Suyash Dubey ${ }^{4} \cdot$ Preeti Gurjar $^{1} \cdot$ Ankit Pandey $^{5} \cdot$ Sivakumar Beena $^{6}$. \\ Ganesh Koneru ${ }^{7}$
}

Received: 2 July 2021 / Accepted: 21 September 2021 / Published online: 9 November 2021

(c) The Author(s), under exclusive licence to Springer Science+Business Media, LLC, part of Springer Nature 2021

\begin{abstract}
The current pandemic of Severe Acute Respiratory Syndrome Coronavirus-2 (SARS-CoV-2) is a global, unanticipated public health crisis. Another emerging challenge is the prevalence of "black fungus", or mucormycosis, among patients who recovered from COVID-19 infection. A retrospective study was conducted on 12 patients in a post-COVID recovery phase who developed mucormycosis. The study parameters evaluated lymphocyte count, CD4+ T cell status, and associated systemic co-morbidities for the patient, namely diabetes. The interventions during the treatment for COVID were also recorded to include administration of oxygen, ventilator assistance (invasive and non-invasive)/oxygen support, and steroid use. The possible relationship between low lymphocyte and CD4+ counts with diabetes and fungal growth was evaluated. It was observed that the majority of the patients who had a positive history for diabetes with low lymphocyte and CD4+ counts were more susceptible to opportunistic fungal infections. Most of the patients, but not all, had a history of receiving oxygen or assisted ventilation, as well as steroids, during the treatment for COVID infection. These interventions may be considered as accessory contributing factors for fungal infection. Post-exposure to SARS-CoV-2, therapies should be targeted at prevention of functional exhaustion of lymphocytes and maintaining optimal lymphocyte and subset counts in susceptible hosts for the prevention of opportunistic fungal infections. The relationship between functional exhaustion of the lymphocyte, diabetes, and COVID mandates further research.
\end{abstract}

Keywords COVID $\cdot$ SARS-CoV-2 $\cdot$ Fungal $\cdot$ Infection $\cdot$ Mucormycosis $\cdot$ Coronavirus $\cdot$ Lymphocytes $\cdot$ CD4 $\cdot$ T cells

\section{Introduction}

The ongoing pandemic, caused by the novel strain of coronavirus Severe Acute Respiratory Syndrome Coronavirus-2 (SARS-CoV-2), is posing a public health and economic

Darpan Bhargava

emaildarpan@gmail.com

1 Maxillofacial Surgery and TMJ Consultancy Services, H-3/2, BDA Colony, Lalghati, Airport Road, Bhopal, Madhya Pradesh, India

2 Head \& Neck Services, COVID Care Wing, Atal Memorial Cancer Care Hospital, Bhopal, Madhya Pradesh, India

3 Department of Anaesthesiology and Critical Care, Gandhi Medical College and Associated Hospitals, Bhopal, Madhya Pradesh, India challenge globally [1]. A secondary condition arising in India is a large number of post-COVID fungal infections. Fungal spores are present in the environment and humans are often exposed to them. Immune-competent hosts safeguard from these fungal infections through natural defence

4 Atal Memorial Cancer Care Hospital, Bhopal, Madhya Pradesh, India

5 Amrit Dental and Maxillofacial Centre, Bhopal, Madhya Pradesh, India

6 Department Oral and Maxillofacial Surgery, Meenakshi Ammal Dental College and Hospital, Chennai, Tamil Nadu, India

7 Department Oral and Maxillofacial Surgery, Sibar Institute of Dental Sciences, Guntur, Andhra Pradesh, India 
mechanisms. Reports of rhino-orbital cerebral mucormycosis (Fig. 1) are common in immune-compromised hosts in sparse numbers. Even with appropriate treatment, this disease has high morbidity and mortality if not detected early [2].

Mucormycosis represents acute or sub-acute, rapidly progressive infections caused by angio-invasive fungi in the order of Mucorales [3]. A rapid surge in cases of the otherwise rare "black fungus" was observed in India in patients recovered from COVID-19 after the second wave of the viral infection. The diagnosis of mucormycosis may be confirmed microscopically using $\mathrm{KOH}$ mounts to demonstrate broad aseptate or sparsely septate ribbon-like hyphae with right angled branching in a tissue specimen or aseptically aspirated material (Fig. 2). Alternatively, routine stains, such as hematoxylin and eosin (H\&E) stains, may be used to visualize mucor hyphae (Fig. 3). The medical community is considering the increase in fungal infections to be multifactorial, with theorized contributory factors of use of industrial oxygen, compromised oxygen transportation during an emergency, use of steroids to manage the COVID induced cytokine storm, nosocomial fungal exposure due to prolonged hospital stay, or contaminated oxygen humidifiers, tubing and masks. The co-morbidities of a patient also contribute to complications of COVID-19 disease including post-COVID fungal infections.

One aspect that is neglected as a contributing factor of fungal infections is the lymphocyte and lymphocyte subset, including CD4+ and CD8+ T cells, statuses of the patient during the active phase of the SARS-CoV-2 and the postCOVID recovery. Depressed CD4+ T cell subsets of lymphocytes is a known cause for fungal infections in patients with human immunodeficiency virus (HIV) [4]. It is well documented that absolute numbers of lymphocytes, CD4+ T cells, and CD8+ T cells are decreased in almost all COVID19 patients, with significantly lower numbers in patients with severe cases [5].

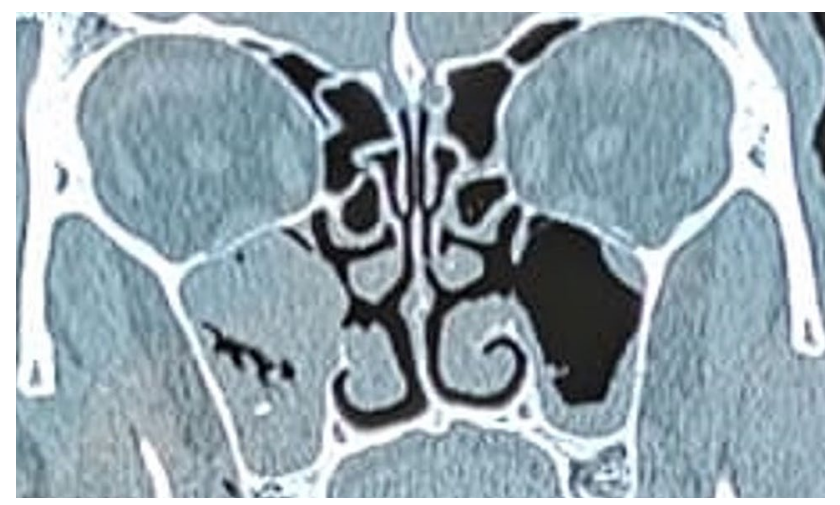

Fig. 1 Computed tomographic (CT) coronal section demonstrating classic mucor growth in the right maxillary sinus

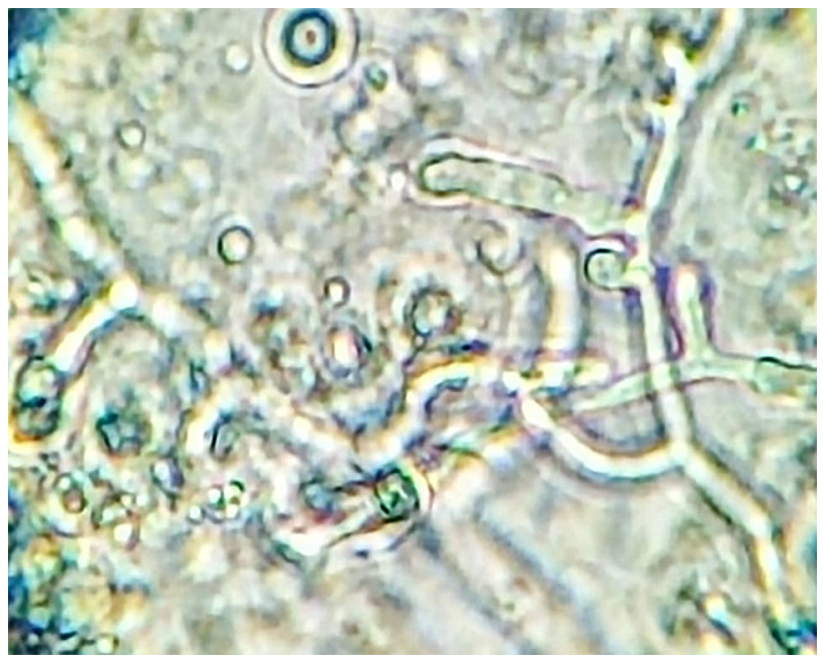

Fig. $2 \mathrm{KOH}$ mount demonstrating mucormycosis with broad aseptate or sparsely septate, ribbon-like hyphae with right-angled branching

From the literature thus far, it may be postulated that reduction and functional exhaustion of lymphocytes (including CD4+ T and other subsets) is the vital contributing factor for the surge in cases of rhino-orbital and cerebral mucormycosis post-SARS-CoV-2 infection.

\section{Materials and Methods}

A retrospective data compilation was done for patients diagnosed with 'mucormycosis' of the maxillofacial region post-recovery from COVID infection. Data was collected on a random selection basis after stratification by disease

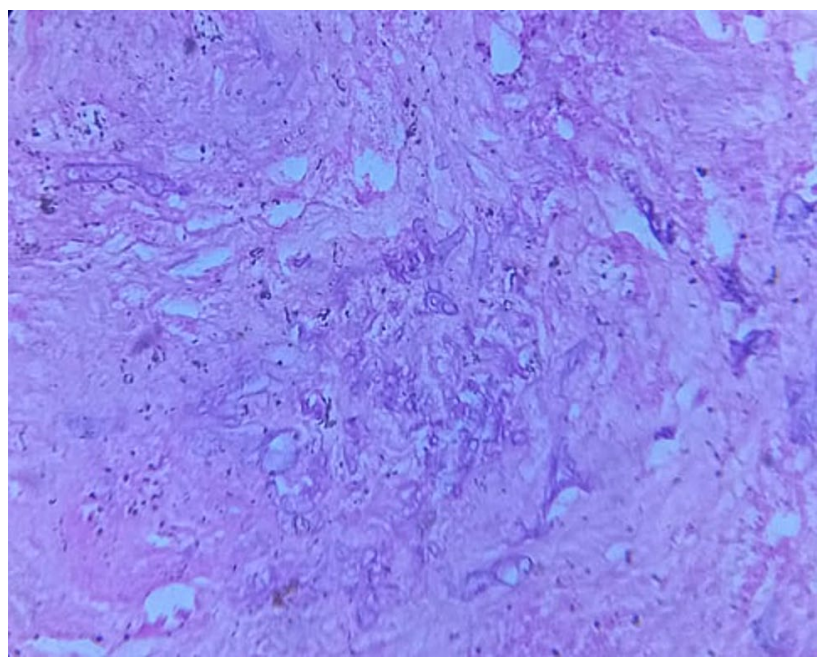

Fig. 3 Hematoxylin and eosin (H\&E) stained specimen, demonstrating mucor hyphae 
severity from the cases reported to the senior consultant and one of the authors of the present paper (DB) for opinion and consultation. All of the patients included for this study had a history of COVID infection that was confirmed using RT-PCR test results. The variables for which data was collected included age and sex of the patient, diabetic status, and treatment for COVID infection including oxygen administration, assisted ventilation, steroid administration, lymphocyte count, and CD4+ T cell count. All data collection was compiled by a single qualified research assistant.

\section{Results}

The observations gathered from the current study indicate that reduced lymphocyte and subset counts qualify as vital contributing factors for development of post-COVID rhinoorbital and cerebral mucormycosis. Twelve study samples were included $(\mathrm{N}=12)$. Among the 12 patients, $8(66.6 \%)$ were males and $4(33.3 \%)$ were females. All the patients had a history of COVID infection preceding mucor infection, confirmed by RT-PCR. Eleven patients (91.6\%) had a history of diabetes. Mucormycosis was diagnosed in the study population after COVID recovery and discharge from the hospital. Four (33.3\%) patients developed mucormycosis less than one week after recovery from COVID, two (16\%) patients in the first week, four (33.3\%) in the second week, and two (16\%) patients were three weeks post-COVID recovery (Table 1$)$.

Only one (8\%) patient did not receive any kind of supportive intervention such as oxygen supplementation or assisted ventilation. The rest of patients, $\mathrm{n}=11(91 \%)$, required oxygen or assisted ventilation. The same eleven patients were also treated with steroids. Eleven patients (91\%) had reduced lymphocyte count for weeks after COVID recovery (Table 1). Eleven patients (91\%) also had reduced lymphocyte count below the normal cut-off value, with only one patient having a lymphocyte count in the normal reference range (at the lower limit side) at the 3rd week post recovery (Fig. 2).

Data was available for two (16\%) patients regarding the CD4 + cell count and it was observed that it was significantly reduced. This suggested a possible correlation between reduced lymphocyte and CD4 + counts, along with co-morbidities, as contributing factors for mucormycosis. The use of medical oxygen and steroids in an immune-compromised host may also pave the way for fungal infections (Table 1; Fig. 4). Out of the 12 study patients, five (41.6\%) fungal infections proved fatal within 2-3 weeks due to cerebral involvement.

\section{Discussion}

The possibility of opportunistic fungal infections in patients recovering from the COVID infection is now documented in the literature. Associated fungal infections are not just restricted to mucormycosis, but include a wide variety of organisms such as invasive aspergillosis, candidiasis, and cryptococcosis. It is essential to assess the risk factors, types of invasive mycosis, appropriate diagnostic methods, and the

Table 1 Summary of post-COVID patients, diagnosed with mucormycotic infection of the maxillofacial region

\begin{tabular}{|c|c|c|c|c|c|c|c|c|}
\hline S. no. & Age & Sex & Diabetic history & $\begin{array}{l}\text { Assisted ventilation/ } \\
\text { supplemental oxygen } \\
\text { support }\end{array}$ & Steroid use & $\begin{array}{l}\text { Establish- } \\
\text { ment of mucor } \\
\text { infection(duration- } \\
\text { post recovery from } \\
\text { COVID) (in week } \\
\text { post recovery) }\end{array}$ & $\begin{array}{l}\text { Lymphocyte count(at } \\
\text { the time of mucor } \\
\text { diagnosis)(\% using } \\
\text { flowcytometry); nor- } \\
\text { mal reference range } \\
20-40 \%\end{array}$ & $\begin{array}{l}\text { CD4+ T cell status(at } \\
\text { the time of mucor } \\
\text { diagnosis) }\end{array}$ \\
\hline 1 & 60 & M & Yes & Yes & Yes & Less than 1 week & 9.6 & NA \\
\hline 2 & 43 & M & Yes & Yes & Yes & Less than 1 week & 06 & 264 \\
\hline 3 & 68 & $\mathrm{~F}$ & Yes & No & No & 2nd week & 02 & NA \\
\hline 4 & 46 & M & Yes & Yes & Yes & 3rd week & 19 & NA \\
\hline 5 & 44 & M & Yes & Yes & Yes & 2nd week & 16 & NA \\
\hline 6 & 27 & M & No & Yes & Yes & 2nd week & 18 & NA \\
\hline 7 & 69 & $\mathrm{~F}$ & Yes & Yes & Yes & 1st week & 09 & NA \\
\hline 8 & 63 & M & Yes & Yes & Yes & 2nd week & 10.5 & NA \\
\hline 9 & 55 & M & Yes & Yes & Yes & 3rd week & 22 & NA \\
\hline 10 & 55 & F & Yes & Yes & Yes & Less than 1 week & 10 & NA \\
\hline 11 & 45 & $\mathrm{~F}$ & No & Yes & Yes & 1st week & 12 & NA \\
\hline 12 & 57 & M & Yes & Yes & Yes & Less than 1 week & 08 & 412 \\
\hline
\end{tabular}

NA data not available 
Fig. 4 The lymphocyte percentage in the patients at the time of diagnosis of mucormycosis infection
Lymphocyte Percentage

Normal reference range: $\mathbf{2 0 - 4 0 \%}$

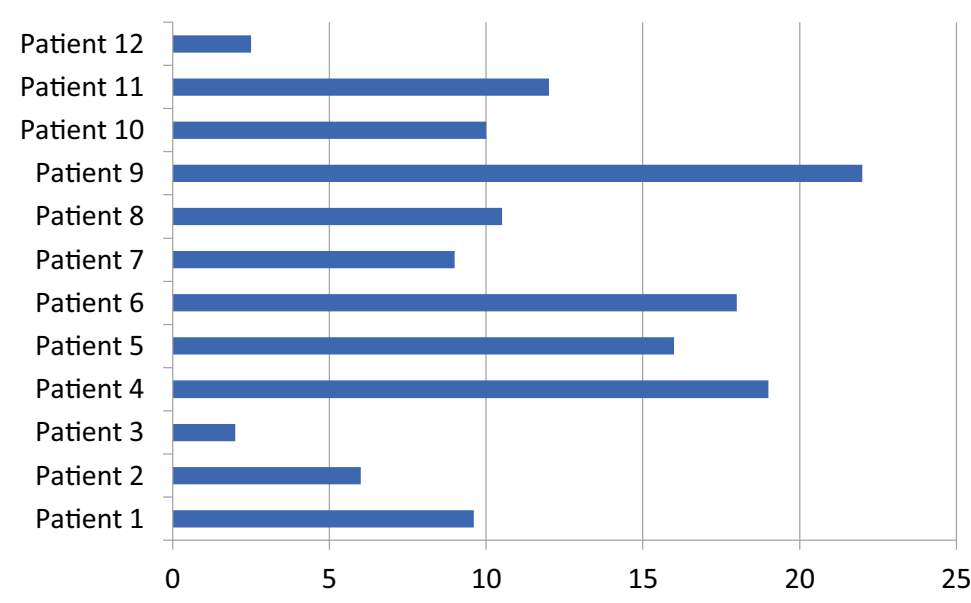

- Lymphocyte count requirement for individual treatment protocols in COVID-19 patients [2].

Diao et al. observed that the number of total $\mathrm{T}$ cells, including CD4+ and CD8+ counts, were intensely reduced in COVID-19 patients, especially in patients admitted to the Intensive Care Unit (ICU). T cells from COVID-19 patients had significantly higher levels of the exhausted marker PD-1, demonstrating functional exhaustion [6]. The absolute number of T lymphocytes, CD4+, and CD8+ T cells were markedly lower in severe COVID-19 cases than moderate cases, and were associated with markedly higher levels of IL-2R, IL-6, IL-10, TNF-alpha, and other inflammatory markers [7].

Liu et al. analysed 95 patients confirmed for COVID with and without diabetes mellitus (DM) and found that lymphocytes and subsets of lymphocytes, especially B and T cells, were reduced in COVID-19 patients, especially in those with DM. Patients with severe COVID-19 and DM had the lowest lymphocyte counts compared to patients with severe COVID-19 without DM and those with non-severe COVID19 with or without DM. Observations indicate that immune system dysfunction or dysregulated host immune response is commonly noted in individuals affected with COVID-19. The authors concluded that the immune-modulatory treatment should be considered to reduce disease progression and prognosis of COVID-19 patients, especially in patients with systemic co-morbidities [8].

Evaluation of dynamic changes in peripheral blood lymphocyte subsets in adult patients with COVID-19 demonstrated that the various lymphocyte subsets $(\mathrm{CD} 3+, \mathrm{CD} 4+$, $\mathrm{CD} 8+, \mathrm{CD} 19+$, and CD16/56+) were below normal ranges at one week after the onset of illness. The lowest point was reached during the second week. The subsets increased gradually during the third week and returned to normal levels in the fifth week, but remained lower than those of the healthy controls. The $\mathrm{CD} 3+, \mathrm{CD} 4+$, and $\mathrm{CD} 8+$ counts were lower in patients with severe disease compared to those with nonsevere disease, and in patients who died compared to those who recovered [9].

Liu D et al. demonstrated that critically ill patients with COVID-19 and DM had lymphocyte and subset counts that were significantly reduced at one week, rapidly elevated to peak at 2 weeks after onset, and then gradually declined during recovery. The COVID-19 critically ill patients with DM had the lowest decline at one week and a slow and lowest rise at two weeks after onset, while COVID-19 non-critically ill patients with DM had the highest rise at two weeks after onset. Both these groups had similar lymphocyte and subset counts at five weeks after onset, which were lower than patients without DM [10]. In addition to the decrease in T cell count, there was a disruption of the $\mathrm{T}$ cell differentiation balance in COVID-19 patients, which affects $\mathrm{T}$ cell function [11].

In a multicenter, retrospective study conducted by Wen et al., the absolute numbers of T-lymphocytes, CD4 + T cells, and CD8+ T cells were decreased in almost all COVID-19 patients, with significantly lower numbers in patients with severe COVID-19 $\left(294.0,177.5\right.$, and $\left.89.0 \times 10^{6} / \mathrm{L}\right)$ than in patients with moderate COVID-19 $(640.5,381.5$, and $254.0 \times 10^{6} / \mathrm{L}$ ). This finding suggested that SARS-CoV-2 infection majorly affects T-lymphocytes, especially CD4+ T cells and CD8 + T cells [12].

The significant reduction of the absolute number of lymphocytes, specifically $\mathrm{T}$ cells, described in the most of the severe COVID-19 cases, may be associated with the worse outcome and a higher risk of developing opportunistic infections. Mucormycosis is a fungal infection caused by a group of opportunistic molds, i.e., mucormycetes [13]. The role of T-cells in invasive mucor as part of the adaptive immune system is highlighted by Potenza $L$ et al. in a brief report on 
a group of haematologically ill patients who suffered from mucormycosis. Their researchers described Mucoralesspecific T-cells (CD4+ and CD8+) that produced cytokines, such as IL-4, IL-10, IL-17 and IFN- $\gamma$, that directly damaged Mucorales hyphae [14]. It is essential to monitor the moderate to severely ill COVID patients with fungal pathogen surveillance through clinical and histopathologic examination, as well as PCR based assays.

Through the observation of this study and previously reported data by various authors, the reduction and the functional exhaustion of lymphocytes (including CD4 + T and other subsets) should be considered as the vital contributing factor for the surge in cases of rhino-orbital and cerebral mucormycosis post SARS-CoV-2 infections, apart from the established predisposing factors that include: ketoacidosis, high blood sugar, iron metabolism related aberrancy, role of ferritin, high serum iron, free radical-induced endothelitis, hepcidin activation, and upregulation of glucose receptor protein (GRP78) [15].

\section{Conclusions}

With the limitation of our small sample size, the relation between functional exhaustion with persistently reduced lymphocytes and subset counts in SARS-CoV-2 in diabetics and vulnerability to post COVID rhino-orbital and cerebral mucormycosis should be considered as a vital parameter for further research. In patients post- SARS-CoV-2 who experienced moderate to severe symptoms, future research, interventions, and therapies should be targeted at prevention of functional exhaustion of the lymphocytes and maintaining the optimal lymphocyte and subset counts with intended effort to prevent opportunistic fungal infections in susceptible hosts. Furthermore, aseptic hygiene protocols are required for the care of such patients, as endorsed through observations of this study and the previously reported data.

Acknowledgements Dr. Rashmi Nichlani, Chief-Histopathologist, SV Diagnostic Centre, Bhopal, Madhya Pradesh, India, for interpreting clinical data and providing photomicrographic records for the study.

Author Contributions Research concept; Dr. Darpan Bhargava MDS, MOMS RCPS(Glasg), PGDHM, Ph.D, Professor, Oral \& Maxillofacial Surgery, People's University, Bhopal, Madhya Pradesh, India.

Funding Self-financed.

Data Availability Included in master table in the presented work.

Code Availability NA.

\section{Declarations}

Conflict of interest None.
Consent to Participate NA.

Consent for Publication NA.

Ethical Approval AMCR/OMFS/2021/A01 (approved).

\section{References}

1. Mittal A, Manjunath K, Ranjan RK, Kaushik S, Kumar S, Verma V. COVID-19 pandemic: insights into structure, function, and hACE2 receptor recognition by SARS-CoV-2. PLoSPathog. 2020;16(8):e1008762.

2. Song G, Liang G, Liu W. Fungal co-infections associated with global COVID-19 pandemic: a clinical and diagnostic perspective from China. Mycopathologia. 2020;185(4):599-606. https://doi. org/10.1007/s11046-020-00462-9.

3. Ajith Kumar AK, Gupta V. Rhino-orbital cerebral mucormycosis. [Updated $2021 \mathrm{Feb} 8$ ]. In: StatPearls [Internet]. Treasure Island, FL: StatPearls Publishing; 2021 Jan. https://www.ncbi.nlm.nih. gov/books/NBK557429/.

4. Holmberg K, Meyer RD. Fungal infections in patients with AIDS and AIDS-related complex. Scand J Infect Dis. 1986;18(3):17992. https://doi.org/10.3109/00365548609032326.

5. Wen X, Jiang D, Gao L, et al. Clinical characteristics and predictive value of lower CD4+ $\mathrm{T}$ cell level in patients with moderate and severe COVID-19: a multicenter retrospective study. BMC Infect Dis. 2021;21:57. https://doi.org/10.1186/ s12879-020-05741-w.

6. Diao B, Wang C, Tan Y, Chen X, Liu Y, Ning L, Chen L, Li M, Liu Y, Wang G, Yuan Z, Feng Z, Zhang Y, Wu Y, Chen Y. Reduction and functional exhaustion of $\mathrm{T}$ cells in patients with coronavirus disease 2019 (COVID-19). Front Immunol. 2020;1:827. https://doi.org/10.3389/fimmu.2020.00827.

7. Gangneux JP, Bougnoux ME, Dannaoui E, Cornet M, Zahar JR. Invasive fungal diseases during COVID-19: we should be prepared. J Mycol Med. 2020;30(2):100971. https://doi.org/10. 1016/j.mycmed.2020.100971.

8. Liu D, Wang Y, Zhao B, Lan L, Liu Y, Bao L, Chen H, Yang M, Li Q, Zeng Y. Overall reduced lymphocyte especially T and B subsets closely related to the poor prognosis and the disease severity in severe patients with COVID-19 and diabetes mellitus. DiabetolMetabSyndr. 2021;13(1):5. https://doi.org/10.1186/ s13098-020-00622-3.

9. Deng Z, Zhang M, Zhu T, Zhili N, Liu Z, Xiang R, Zhang W, Xu $Y$. Dynamic changes in peripheral blood lymphocyte subsets in adult patients with COVID-19. Int J Infect Dis. 2020;98:353-8. https://doi.org/10.1016/j.ijid.2020.07.003.

10. Liu D, Lan L, Luo D, Zhao B, Wei G, He Y, Zhang R, Liu Y. Lymphocyte subsets with the lowest decline at baseline and the slow lowest rise during recovery in COVID-19 critical illness patients with diabetes mellitus. Diabetes Res Clin Pract. 2020;167:108341. https://doi.org/10.1016/j.diabres.2020.108341.

11. Liu L, Xu L, Lin C. T cell response in patients with COVID-19. Blood Sci. 2020;2(3):76-8. https://doi.org/10.1097/BS9.00000 00000000050.

12. Wen X, Jiang D, Gao L, et al. Clinical characteristics and predictive value of lower CD4+ $\mathrm{T}$ cell level in patients with moderate and severe COVID-19: a multicenter retrospective study. BMC Infect Dis. 2021;21:57. https://doi.org/10.1186/ s12879-020-05741-w.

13. Pasero D, Sanna S, Liperi C, et al. A challenging complication following SARS-CoV-2 infection: a case of pulmonary 
mucormycosis. Infection. 2020;10:20. https://doi.org/10.1007/ s15010-020-01561-x.

14. Potenza L. Vallerini D, Barozzi P, et al. Mucorales-specific T cells emerge in the course of invasive mucormycosis and may be used as a surrogate diagnostic marker in high-risk patients. Blood. 2011;118:5416-9.

15. Jose A, Singh S, Roychoudhury A, Kholakiya Y, Roychoudhury Arya S, S,. Current understanding in the pathophysiology of SARS-CoV-2-associated rhino-orbito-cerebral mucormycosis: a comprehensive review. J Maxillofac Oral Surg. 2021;10:20. https://doi.org/10.1007/s12663-021-01604-2.

Publisher's Note Springer Nature remains neutral with regard to jurisdictional claims in published maps and institutional affiliations. 